\title{
Mercureid Gives New Opportunities for Treatment and Prevention of Complications Caused by COVID-19 in Patients with Ophthalmic Pathologies
}

\author{
Sergey $\mathrm{N}_{\text {Gusev }}{ }^{*}$, Velichko LN², Bogdanova $\mathrm{AV}^{2}$, Khramenko $\mathrm{NI}^{2}$, Konovalova $\mathrm{NV}^{2}$, Kovtun $\mathrm{AV}^{3}$ \\ ${ }^{1} \mathrm{PhD}$ in Medicine and Health Sciences, Author and Owner of Intellectual Property Rights for New Mercureid \\ Molecules, Ukraine \\ ${ }^{2}$ The Filatov Institute of Eye Diseases and Tissue Therapy of the National Academy of Medical Sciences of \\ Ukraine, Odessa, Ukraine \\ ${ }^{3}$ Assistant of the Department of Ophthalmology, Odessa National Medical University, Ukraine \\ *Corresponding Author: Sergey N Gusev, PhD in Medicine and Health Sciences, Author and Owner of \\ Intellectual Property Rights for New Mercureid Molecules, Ukraine; Email: mercurid@te.net.ua
}

Received Date: 13-09-2021; Accepted Date: 18-10-2021; Published Date: 26-10-2021

Copyright $^{\odot} 2021$ by Gusev SN, et al. All rights reserved. This is an open access article distributed under the terms of the Creative Commons Attribution License, which permits unrestricted use, distribution and reproduction in any medium, provided the original author and source are credited.

\begin{abstract}
The coming era of COVID-19 introduces critical challenges for researchers. Accordingly, new questions connected with the current state of people's health arise, namely, what consequences will be for the patients who have suffered from long COVID-19; what diseases are supposed to threaten the patients in the future; what immunity responses will be after the influence of antigen SARS-CoV-2; to what extent the organism changes caused by SARS-CoV-2 contribute to or prevent from the development of long-term protective immunity; how effective COVID19 vaccine will be for these patients.

In this connection, some of the above-mentioned items have been highlighted in this research work.

The study was conducted in 49 patients with ophthalmic pathologies who had previously undergone COVID-19. It should be stressed that examination of eye disorders is very essential because they allow in a non-traumatic way to obtain lifetime visualization of the state of blood vessels and capillaries, as well as to assess the effect of the virus on the central nervous system. Moreover, immunological studies made it possible to draw a conclusion about the state of
\end{abstract}


antiviral immunity, immune status and its correlation with the severity of inflammatory processes in the eye structure, the central nervous system including the vascular endothelium.

The novelty of the study is in establishing a causal relationship between SARS-CoV-2 infecting and formed dysfunction of immune parameters that caused the manifestation of chronic inflammatory diseases. As a result, light adaptation was impaired by 2.3 times, due to the damage to blood circulation owing to the neurotoxic effect of SARS-CoV-2 and hypoxemia. The corrective effect of drug Mercureid was registered in $73.4 \%$ of patients.

It should be indicated that the most serious cases were observed in the group of patients with the damage to the retinal vascular system: the phenomenon of re-thrombosis of both the central retinal vein and its branches, as well as circulatory disorders in the optic nerve trunk - ischemic optic neuropathy with a sharp deterioration in vision. In these patients, the combination of vascular drugs and drug Mercureid allowed stabilizing the patients' state, achieving remission and in $50.0 \%$ of cases having higher visual functions.

Firstly, Mercureid made venotonic and angioprotective effect. It reduced vein elasticity and capillary permeability. Moreover, it improved venous outflow and microcirculation that allowed in some cases to restore lymphatic drainage.

According to the results of the immune study, the targeted effect of the new drug Mercureid, aimed at modulating the activity of several critical target proteins, such as CD3, CD4, CD8, CD25, CD38, CD54, CD95 was revealed. The therapeutic efficacy of Mercureid was $75.1 \%$.

The second research finding is that in patients with manifestation of chronic inflammatory diseases who have previously been infected with SARS-CoV-2, the production of specific protective antibodies is supposed to be impaired (as these patients often have pathologically low levels of CD4, CD8, CD25 and overexpression of CD38, ICAM-1, CD95 that, consequently, causes apoptosis of immune cells, lymphopenia and also forms the phenotype of exhausted T-cells with activation of the expression of inhibitory receptors). Thus, we consider vaccination to be ineffective for them, due to the presence of a compromised immune system. Accordingly, the provision of corrective multitarget immunotherapy aimed at several target proteins, which are critical for the formation of long-term effective post-viral immunity to SARS-CoV-2, is certainly an important therapeutic need. This immunotherapy can be carried out both before and after vaccination in order to achieve the maximum protective effect from the vaccine. However, the definite answer to this research question requires another type of study design, which is under our further consideration.

\section{Keywords}

Immunotherapy; SARS-Cov-2; COVID-19; Ophthalmology 


\section{Introduction}

One of the most pressing problems of medicine in the modern world connected with the mankind survival is the high rate of morbidity and mortality from viral infections, which are widespread in the human population, capable of affecting almost all organs and systems of the human's body. We should keep in mind that over the past decades, humanity has experienced serious trials. There have never been such rapid pandemics in the world history, which during a short period of time took away many lives not only of people suffering from chronic diseases, but also of those people who, before the disease, considered themselves practically healthy, regardless of age, gender, social and material status in the society. Moreover, viral infection is a cofactor for the progression of HIV infection and AIDS (Isakov VA, et al., 2006; Samgin MA, Khaldin AA, 2002). Regretfully, the lessons from past pandemics have taught little to humanity.

\section{SARS-CoV-2}

Since December 2019, COVID-19 has evolved into a global pandemic of severe acute respiratory syndrome caused by a new highly transmissible RNA virus identified as a member of the family of Coronaviridae. SARS-CoV-2 is a beta coronavirus. It enters the cells of the human body using two proteins: the first one is ACE2 cell receptor (Angiotensin-Converting Enzyme 2), connected with the angiotensin converting enzyme and the other is TMPRSS2 enzyme.

It is worth mentioning that the ophthalmologist was the first to report the virus in Wuhan. He himself contracted and died from the disease while treating a patient for glaucoma. Ophthalmic manifestations vary in form, severity and timing. $\mathrm{Wu}$, et. al., suggested that ophthalmic manifestations are more common in patients with severe systemic diseases with abnormal blood parameters and inflammatory processes [1]. It is obvious that on being infected with SARS-Cov-2 virus, which causes COVID-19, the respiratory tract, intestines, kidneys and eyes are endangered first of all, because two proteins necessary for the virus are present in these organs. Even in the study of atypical pneumonia SARS and Middle East Respiratory Syndrome (MERS), it was found out that coronaviruses are able to penetrate through nerve cells into the brain.

MRI studies have shown an increase in the signal from the white matter of the posterior rectus gyrus in the cerebral hemispheres and from the olfactory bulbs. Taking into account the study of the patients ill with COVID-19, we have come to the conclusion that the virus causes changes in white matter only during the first days of infection and in some patients, respiratory failure may appear due to the effect of the virus on the brain structures that are part of the limbic system. However, the organ of vision is also affected, because ACE2 receptor is known to be widely expressed in many tissues, including the retina, which is an extension of the central nervous system. It should be noted that ACE2 receptor is involved in the pathogenesis of 
diabetic and hypertensive retinopathy. Expression of ACE2 has also been found in the cornea, human aqueous humor and retinal tissue, pigment epithelial cells, photoreceptors and Müller cells. In addition, coronaviruses are known to cause inflammation in animals directly in the organ of vision, including retinitis and optic neuritis.

According to meta-analysis 2020, among patients with COVID 19, the overall prevalence of eye disoders is estimated at $7 \%$ (95\% CI: $0.03-0.10)$. It is known that conjunctivitis can be the entrance gate of infection [2]. The researchers also state that the spread of the virus in the conjunctiva may persist even after a nasopharyngeal swab becomes negative for SARS-Cov-2 virus [3].

Conjunctivitis is the most common ophthalmic manifestation which is found in patients with COVID-19. In a large series of cases with mild COVID-19 infection, Sindhuja, et al., reported that $8.66 \%$ of patients had conjunctivitis. All symptomatic patients had a history of redness in one or both eyes. Respiratory tract symptoms have been connected with conjunctival congestion.

\section{Conjunctivitis in Children}

A 30-time increase in children's morbidity of Kawasaki disease has been reported in some parts of Italy that have the highest number of COVID-19 cases. This atypical manifestation is known as Multisystem Inflammatory Syndrome in Children (MIS-C) [4]. Kawasaki disease, a form of self-limiting vasculitis, is connected with iridocyclitis, punctate keratitis, vitreous opacity, papilloedema edema and subconjunctival hemorrhage [5]. In the available literature on MIS-C, ophthalmic manifestations have been predominantly in the form of conjunctivitis.

\section{Pathogenesis of COVID-19}

According to the data of the world researches, there are three main COVID-19 pathogenetic mechanisms which are described as: inflammatory syndrome as a result of viral invasion; proinflammatory state with hypercoagulability and "cytokine storm"; hypoxia and hypertension. Virus damage to endothelial cells via ACE-2 receptor has been shown in the lungs, heart, kidneys, intestines and brain. Histopathological studies have demonstrated direct viral invasion of endothelial cells, endotheliitis and vasculitis in both arterial and venous beds. Inflammation of endothelial cells causes edema, blockage and thrombosis of small vessels that ultimately leads to organ ischemia [6,7]. One theory of hypercoagulation associated with COVID-19 suggests that SARS-CoV-2 promotes the recruitment of inflammatory cells into the blood vessels, resulting in the release of inflammatory markers and cytokines, which subsequently activate the coagulation cascade [8].

The clinical results show a significant increase in prothrombin time, high D-dimer levels and increased concentrations of proinflammatory cytokines and inflammatory biomarkers in

Gusev SN | Volume 2; Issue 3 (2021) | JOAR-2(3)-024 | Research Article

Citation: Gusev SN, et al. Mercureid Gives New Opportunities for Treatment and Prevention of

Complications Caused by COVID-19 in Patients with Ophthalmic Pathologies. J Ophthalmol Adv Res.

2021;2(3):1-17.

DOI: http://dx.doi.org/10.46889/JOAR.2021.2305 
patients with more complicated progression of the disease, indicating the probability of disseminated intravascular coagulation or thrombotic microangiopathy [9].

COVID-19 patients suffer from moderate to severe hypoxemia, which can contribute to dysfunction of many organs, especially the central nervous system. Hypoxemia causes functional changes in ion channels sensitive to oxygen, the $\mathrm{Na}+\mathrm{K}+-$ pump, disrupts the processes of excitation and inhibition of neuronal and glial cells, activates excitotoxicity [10].

In COVID-19 patients, ACE-2 receptor function is exhausted immediately after binding to SARS-CoV-2. There is an increase in Ang II - type II angiotensin and a decrease in the levels of Ang 1-7 (angiotensin 1-7) in tissues that leads to an aggravation of hypertension [11].

Currently, it has been suggested that antihypertensive drugs, the effect of which is based on inhibiting ACE or blocking ACE-2 receptor, can either aggravate or, conversely, improve the clinical course of COVID-19 [12].

COVID-19 has a wide range of clinical manifestations, from asymptomatic to symptomatic disease progression. Infection with the virus causes latent, acute, chronic and slow forms of infection. The immune response of the human body to a viral infection is divided into two phases - the phase of localization of the virus in a limited anatomical area and the phase of late specific influence, during which the localized infection is removed.

In COVID-19 patients other infections (viral, bacterial, fungal) arising together with COVID disease were often found [13].

Thus, a positive test result for another pathogen does not exclude infection with COVID-19 and vice versa. The prevalence of acute acquired concomitant or secondary infections arising in the context of COVID-19 is not precisely determined, but, apparently, it is low and it depends on local factors, including the presence of endemic and new infections in the region [14-18].

It should be stressed that overuse of antibiotics is one of the contributing factors to the disease. It increases the risk of the emergence and spread of multidrug-resistant bacteria. Infections caused by these bacteria are more difficult to treat and they increase morbidity and mortality.

\section{COVID-19 and Ophthalmology}

According to the recent data from the research literature, it is known that:

1. The full range of clinical manifestations of ophthalmic pathologies connected with COVID-19 is not completely cleared out; new clinical symptoms are still being described

2. Structural and functional changes in the organ of vision both as a result of the direct viral effect of COVID-19 and as a result of a violation of homeostasis are still being studied

3. Some researchers suggest that post-COVID syndrome and disorders in the visual analyzer are considered as an underestimated large-scale problem [19] 


\section{Post-COVID Syndrome in Ophthalmology}

All of the above-mentioned factors in the pathogenesis of COVID-19 and post-COVID syndrome are also relevant for ophthalmology. The global coronavirus pandemic has farreaching and long-term consequences. The full spectrum of the disease is still under examination. The disease consequences for various organs, multiple views, theories of pathogenesis and associations with SARS-CoV-2 severe acute respiratory syndrome are under urgent examination today.

The search for term "COVID-19" in search engines offers about a hundred thousand articles. To specify this term by adding the word "ophthalmology", the results are less numerous, but there are still about 1000 publications. To limit the search, the words "ophthalmic manifestations" are introduced. They are met about 100 times.

The literature search was carried out in PubMed for the terms COVID-19, SARS-CoV-2, "ophthalmology", "ophthalmic manifestations", "anterior segment", "conjunctiva", "eye surface", "retina", "vascular membrane of the eye", "uveitis", "neuro-ophthalmology". The articles in English published from January 1, 2020 to January 31, 2020 were under analysis to summarize the current understanding of the ophthalmic manifestations of SARS-CoV-2 virus. 46 case reports, 11 crossover / cohort observational studies, 5 prospective interventional studies, 3 animal models / autopsies and 6 reviews / meta-analyzes were reviewed.

\section{Ophthalmotoxicity of Drugs Used to Treat COVID-19}

Medicines used to treat COVID-19 are toxic to the eyes. Long-term use of chloroquine and hydroxychloroquine may result in retinal toxicity. Medicines Lopinavir / ritonavir may cause reactivation of autoimmune diseases. Ribavirin is less frequently used to treat COVID-19, because it is known to cause retinopathy, retinal vein occlusion, serous retinal detachment, nonarterial ischemic optic neuropathy and Vogt-Koyanagi-Harada disease (VKH). Interferon was connected with retinopathy, conjunctivitis, uveitis, optic neuropathy, corneal ulcers, epithelial defects and Sjogren's syndrome. It is reported that tocilizumab causes retinal hemorrhages. Systemic corticosteroids are known to cause cataracts, glaucoma and central serous chorioretinopathy. These points should be taken into account by an ophthalmologist during the history, examination and treatment of patients [20].

Therefore, it is important for ophthalmologists to know not only about the ophthalmological manifestations of a new viral infection in order to diagnose correctly, but also treat with drugs that have a high profile of toxicological safety and therapeutic efficacy, but their pharmacological properties were unknown to physicians. 


\section{Innovations in the Treatment of Post-COVID-19 Syndrome}

The search for new opportunities in the treatment and prevention of complications caused by COVID-19 is undoubtedly an urgent challenge of the present time. Our attention was paid to a new original drug that has no world analogues - Mercureid, a targeted (targeted action) drug aimed at reducing the overexpression of the proinflammatory cytokine $\mathrm{TNF} \alpha$ and normalizing the production of the most important, if not the main immunological factor of mucosal immunity (mucosalimmunity)-SIgA [21].

Swiss molecular biologist V. A. Loroch (Ph.D. in Biology, Experimental research strategies and research methods in molecular genetics and molecular biology, Switzerland), said that Mercury's interaction with the immunoglobulin superfamily changes the paradigm of our thinking about the mode of action of a drug, as it leads to multiple mechanical biological responses (available at the link https://vimeo.com/435729749):

1. Stimulation of secretory immunoglobulin A. Secretory IgA on the surface of the mucous membrane of the respiratory tract prevents the adhesion of viruses to the surface of the epithelium. Secretory IgA has a non-inflammatory protective function: antibodies of this type can bind to the virus without activating complement and without releasing inflammatory mediators

2. Stimulation of neutralizing immunoglobulin G. Neutralizing IgG prevents the systemic spread of the virus

3. Activation of CD4 +, CD8 + and CD16 + T-cells, which kill virus-infected cells

4. Activation of macrophages. Macrophage-driven phagocytic elimination of apoptotic cells is an immunologically silent response; there is no inflammation. In fact, it is antiinflammatory because macrophages consume apoptotic neutrophils and other cells that produce and secrete pro-inflammatory cytokines as a result of viral infection [22-24]

Thus, we can state that after long COVID-19, a negative PCR test is only the beginning of a difficult path to complete recovery. And in $61 \%$ of patients, the signs of post-COVID syndrome are kept for a long time [25].

\section{Purpose of the Study}

- To investigate the functional state of the Visual Analyzer (VA), regional hemodynamics and immune status in patients after suffering COVID-19 and patients with post-COVID syndrome complicated by ischemic optic neuropathy, as well as the possibility of correction effect in the course of therapy with Mercureid on the identified disorders

- To study the level of expression of apoptosis markers (CD 95), intercellular adhesion ICAM-1 (CD54), as well as CD3, CD4, CD8, CD25, CD38 in healthy individuals and in the patients who have undergone COVID-19 
In respect that while searching the information on the topic: "the level of expression of markers of apoptosis (CD95), intercellular adhesion ICAM-1 (CD54), CD3, CD4, CD8, CD25, CD38 in patients undergoing COVID-19" we haven't found any information available to the public, this immunological study provides a new and important material for practitioners and scientists, allowing for better understanding of the immunopathogenesis of COVID-19.

\section{Materials and Methods}

This study is open, according to the declaration of Helsinki Ethical Principles for Medical Research, the Council of Europe Convention on Human Rights and Biomedicine, as well as the relevant laws of Ukraine.

The studies were carried out with the informed consent of the patients who were examined and treated in the Department of Inflammatory Eye Pathology of the State Institution "The Filatov Institute of Eye Diseases and Tissue Therapy of the National Academy of Medical Sciences of Ukraine".

Ministry of Health of Ukraine, Odessa National Medical University, Commission on Bioethics No. IRB 00004535 Odessa Ste Med U IRB \# 1. Protocol of the meeting of the commission on bioethics №11 from 09.12.2019.

All patients had a history of COVID-19 about 2-3 months before the examination. At the time of treatment and examination, all SARS-CoV-2 IgM antibodies were negative.

\section{Study Population}

Under our control there were 49 patients who had undergone COVID-19. They were divided into three groups.

Group 1 included 17 patients who had undergone COVID-19 and did not have eye diseases (a group of volunteers) who received an outpatient examination and drug correction.

Group 2 included 18 patients with high-risk chronic eye diseases: with posterior uveitis - focal chorioretinitis (7 patients) and disseminated chorioretinitis (11 patients) of various etiology.

Group 3 included 14 patients with ophthalmopathology of vascular genesis: 9 patients with thrombosis of the central retinal vein and its branches and 5 patients who received eye complications after COVID-19 disease, but who hadn't had previous eye pathology. These 5 people were admitted with a diagnosis of acute vascular optic neuropathy in both eyes.

The average age of the patients was $30 \pm 19.5$ (from 30 to 50 years old). The average gap between the symptoms of COVID-19 manifestation and ophthalmic pathology manifestation was 5.6 (average $4.2 \pm 8.2$ ) days. 
Control group - 53 practically healthy people of the control group (age 31 $\pm 20,4$ years old).

All patients were examined according to the usual procedure: examination of visual acuity, visual field, intraocular pressure, ultrasound diagnostics, biomicroscopy, ophthalmoscopy, photo registration. The examination of light sensitivity (SP) of the central zone of the retina was carried out on a semi-Automatic-Recording Visual Adaptometer ARP-1. The procedure is the following. The examination is carried out for 10 minutes, 3 of which - light adaptation, 7 minutes - dark adaptation. Light adaptation occurs when the patient's gaze is fixed in a light sphere (illumination $1200 \mathrm{~cd} / \mathrm{m} 2$ ), then the patient is shown test objects - a cross, a circle, a square. The researcher varies the illumination limits of these objects from $2 \times 108$ to $7.0 \mathrm{~cd} / \mathrm{m}^{2}$. The lowest illumination of an object that the patient notices is marked as a result. The examination is carried out during the first seconds after light adaptation, then it is done at the $2^{\text {nd }}$ minute, $4^{\text {th }}$ minute, $6^{\text {th }}$ minute and $7^{\text {th }}$ minute.

Rheoophthalmography (ROG) and rheoencephalography (REG) are done by a computer rheograph device Reokom (Kharkov, Ukraine).

The immunological study was carried out in the laboratory of immunology of the State Institution "The Filatov Institute of Eye Diseases and Tissue Therapy" and it was aimed at the determination of the immune status, including the determination of antigens of the eye structure and the expression of biomarkers such as: markers of T-lymphocytes CD3, T-helper CD4, cytotoxic T-cell antigen CD8, Interleukin-2 receptor alpha chain (also called CD25), activator of B-cells and T-cells - CD38; ICAM-1 (Intercellular Adhesion Molecule 1) also known as CD54, Fas/CD95 death receptor. They were determined by the immunohistochemical method in 49 patients (the average age of patients is $30 \pm 19.5$ (from 30 to 50 years old)) within 1-2 months after recovery from COVID-19 and in 53 practically healthy people of the control group (age $31 \pm 20,4$ years old).

The patients of the first group were taking Mercureid as monotherapy for 1 month. The patients of the second and third groups were undergoing a course of traditional anti-inflammatory therapy with Mercureid taking for a 14-day period and subsequently they had a course of Mercureid as monotherapy which lasted 1 month.

\section{Statistical Analysis}

Statistical analysis was carried out by means of STATISTICA 8.0 application (StatSoftInc). When analyzing the data, the mean values (M) and the error of the mean $(\mathrm{m})$, Standard Deviation (SD) were taken into account. To determine the paired differences, Student T-test and Wilcoxon test were used. 


\section{Results}

As a result of the virus infection COVID-19, the patients of the first group complained of having blurry spots in front of their eyes, fatigue, dizziness, inability to concentrate and perform complicated tasks. It should be noted that there was also fear of falling ill again. Visual acuity in these patients was not impaired for both long and close distances. The state of the anterior segment of the eyeball in all patients was without pathological changes. Examination of the fundus revealed no gross pathological changes, except for narrowing of the arteries, plethora and tension of the veins. 4 patients had a slight edema along the upper outer arcade of the arteries, 3 patients had a slight edema along the inferior nasal arcade of the arteries; the veins were tense and full of blood.

The light sensitivity of the photopic afferent system of the visual analyzer of this group of patients was assessed based on the determination of the thresholds of light perception. The weakest light stimulus that causes the sensation of light is called the minimum threshold or absolute threshold of vision. The reciprocal of the absolute light threshold characterizes light sensitivity (log units). We compared SP in these patients, the healthy volunteers and the patients with Hypertonic Disease (HD), 1 stage.

Light sensitivity is the most important and the oldest visual function - to distinguish light from darkness. The process of light sensation is primarily formed at the level of the photosensory layer of the retina. The molecules of visual pigment located in the outer segments of the photoreceptors absorb light quanta and, decomposing, provide a consistent course of photochemical, electrical, ionic and enzymatic processes. During the first minutes after dark adaptation, the patients showed a sharp impairment of this function: light sensitivity is 6-7 times lower than in the patients with hypertension and people with the normal function, at the $2^{\text {nd }}$ minute - 4-5 times lower, at the $4^{\text {th }}$ minute - 2-3 times lower, at the $6^{\text {th }}$ minute - on average 2 times lower, at the $7^{\text {th }}$ minute it was reduced by 1.9 times $(\mathrm{p}<0.05)$ in comparison with the group of patients with hypertension and 2.3 times $(\mathrm{p}<0.05)$ in comparison with control group (Fig. 1). Thus, a sharp decrease in light sensitivity is determined throughout the entire examination in the patients who have undergone COVID-19. It is known that in violation of the architectonics of the retina, ischemic process and metabolic starvation, light sensitivity, as one of the oldest and basic functions of the visual analyzer, suffers first being the most sensitive to changes in trophism. In this case, these indicators show an expressed violation of the trophism of the retina of these patients.

According to the results of the study of ocular blood circulation in the first group of patients, a decrease in the volumetric pulsatile ocular blood flow appropriate to RQ indicator by $10 \%$ ( $p$ $<0.05)$ was revealed, as well as an increase in the tonic properties of large vessels of the eye by $20 \%$ in comparison with the norm ( $p<0.05$ ) (Fig. 2 and 3). These indicators did not have statistical difference from the group of patients with hypertension disease. 


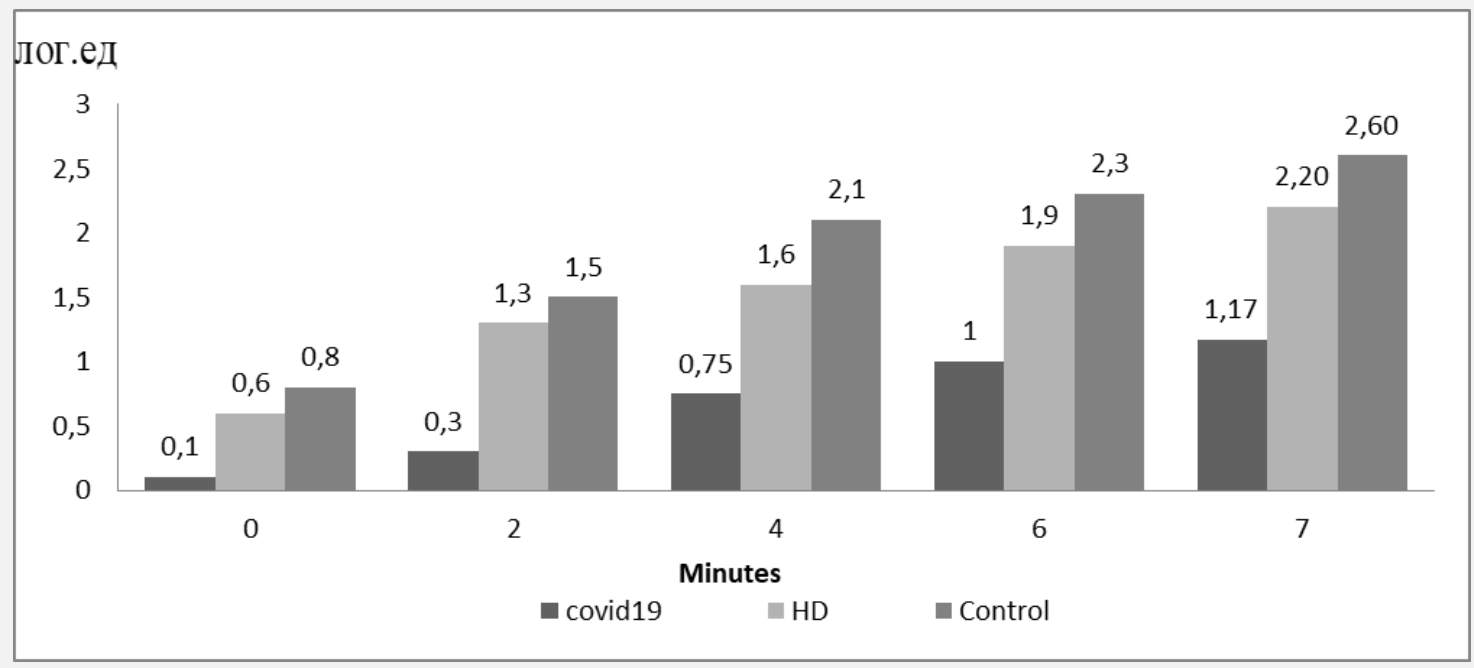

Figure 1: Photopic light sensitivity values (log units) in the patients with long COVID-19, the patients with hypertension disease and the control group (healthy volunteers) at $0,2,4,6$ and 7 minutes of dark adaptation.

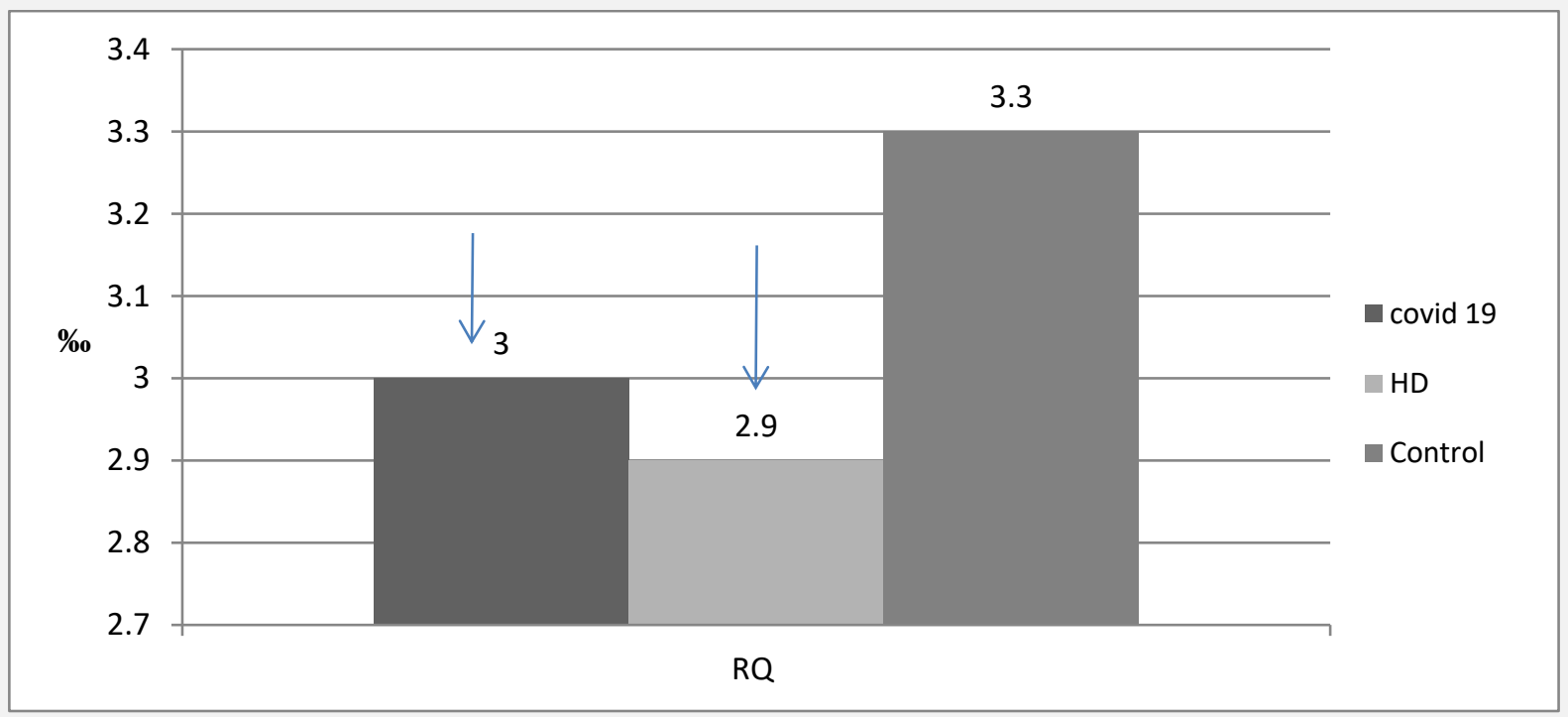

Figure 2: The values of the rheographic coefficient RQ \% (volumetric pulsatile ocular blood flow) in patients with long COVID-19, patients with hypertension disease and control groups (healthy volunteers). 


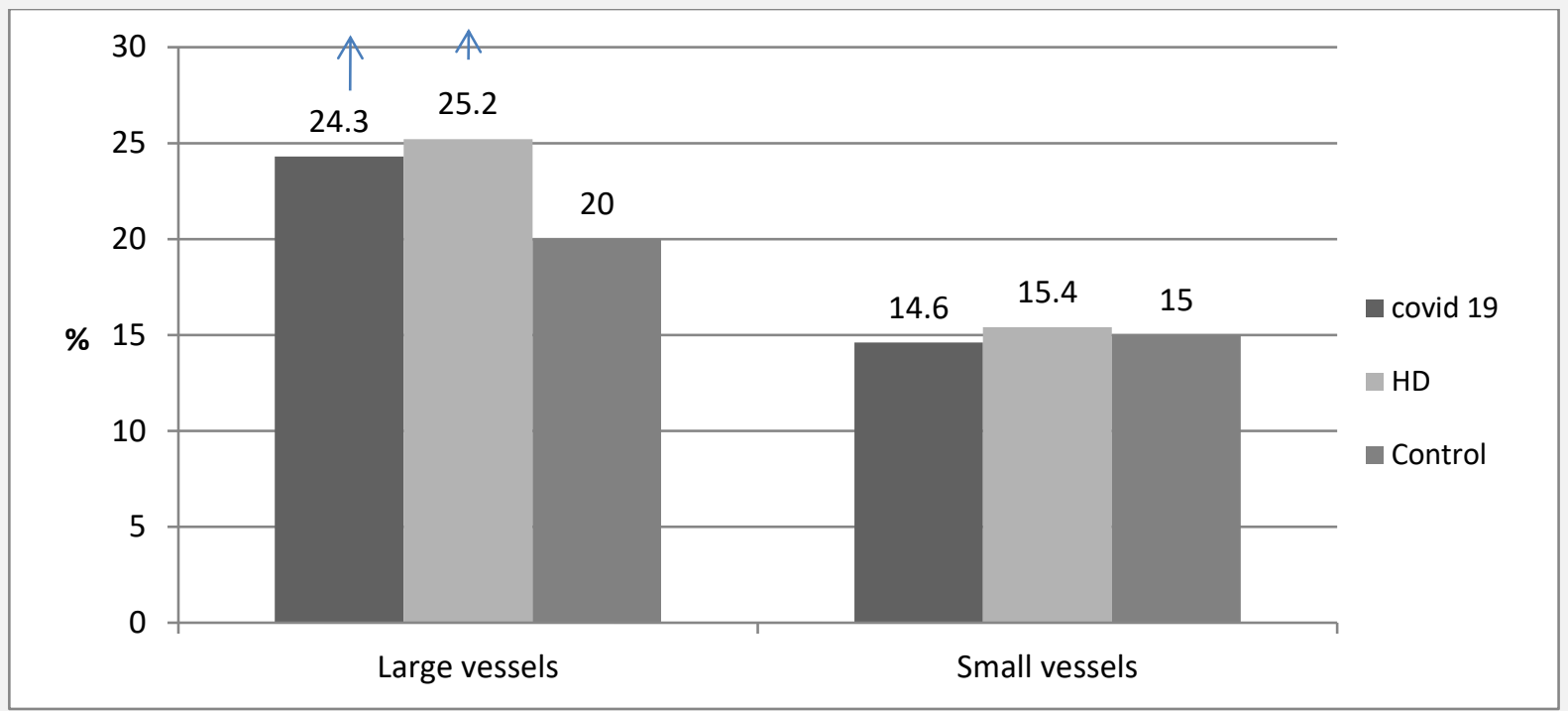

Figure 3: Indicator of elasto-tonic properties of large and small blood vessels $\alpha$ / T (\%) according to rheoophthalmography in the patients with long COVID-19, the patients with hypertension disease and the control group (healthy volunteers).

According to the rheoencephalogram data of the first-group patients, the volumetric pulsatile brain blood flow did not change, but the tonic properties of large (by $54 \%, \mathrm{p}<0.05$ ) and small (by $12 \%, \mathrm{p}<0.05$ ) vessels increased (Fig. 4 and 5).

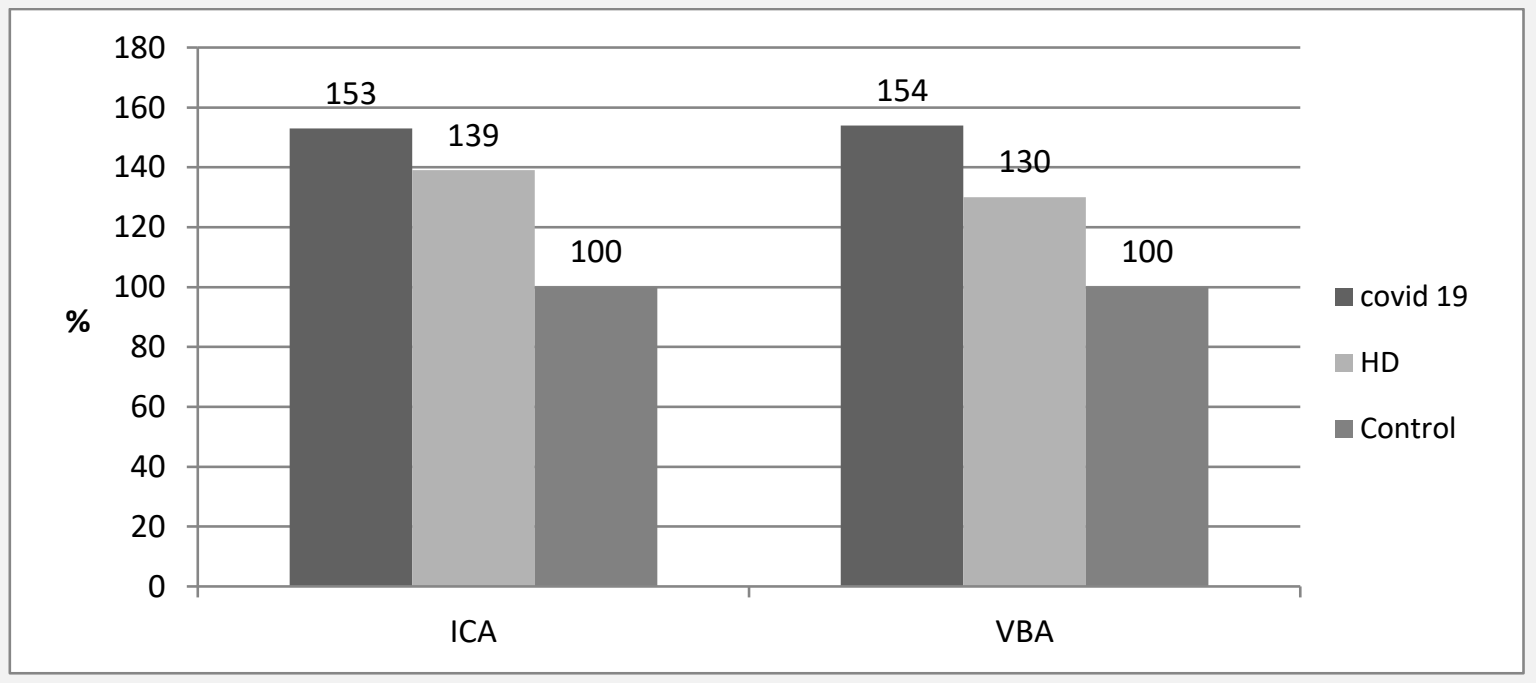

Figure 4: The indicator of the elasto-tonic properties of large blood vessels $\alpha$ / T (\%) in the basins of the Internal Carotid Artery (ICA) and Vertebrobasilar Artery (VBA) according to rheoencephalography in the patients with long COVID-19, the patients with hypertension disease and the control group (healthy volunteers). 


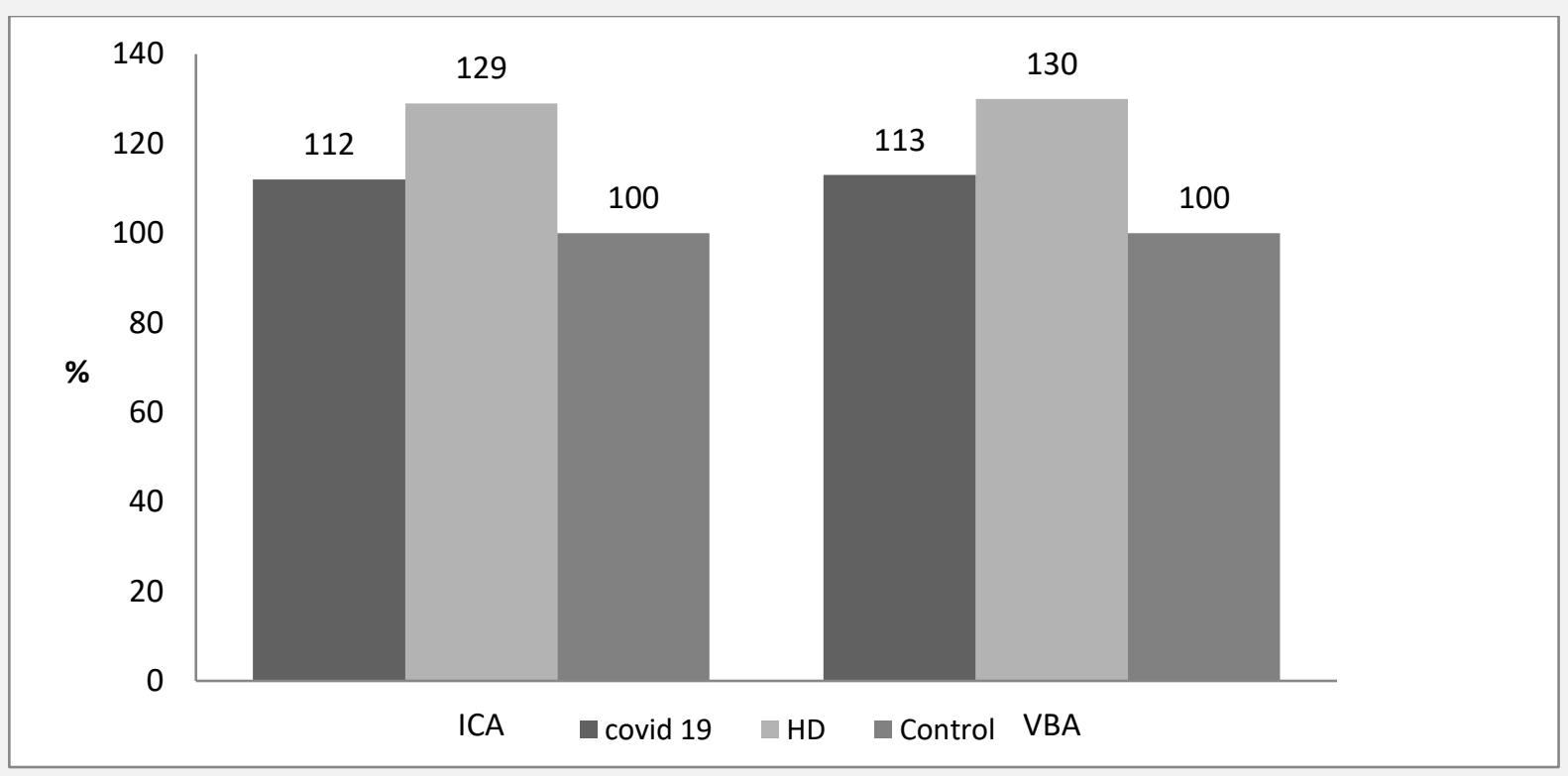

Figure 5: The indicator of the elasto-tonic properties of the slam blood vessels (\%) in the basins of the Internal Carotid Artery (ICA) and Vertebrobasilar Artery (VBA) according to rheoencephalography in the patients with long COVID-19, the patients with hypertension disease and the control group (healthy volunteers).

Thus, the first group of patients in the absence of obvious structural changes in the organ of vision at high resolution (1.0) showed functional disorders in the form of impaired adaptation, which was caused not only by impaired blood circulation both in the eye itself and in the central part of the visual analyzer, but probably, by the neuro-toxic effect of the viral agent itself, hypoxemic disturbance of the homeostasis system. The inclusion of drug Mercureid in one month-therapy program improved the functional state of the retina that resulted in the light sensitivity on average $18 \%$ increase at all minutes of examination $(\mathrm{p}<0.05)$.

The second group of patients who had previously had eye diseases and were in the remission state from 2 to 8 years before COVID-19 disease, complained of decreased visual acuity and appearance of new blurred spots in front of the eye. Out of 7 patients with focal chorioretinitis, 2 people had fresh exudative foci in the central zone that were not connected with the old lesion. 2 patients had juxtapapillary foci with symptoms of exudation at the optic nerve head. 3 patients had exudation along the edge of the old lesion. During the past month, all inflammation phenomena were blocked. Visual acuity in the patients with lesions of the central zone increased slightly against the background of treatment. Despite the complete block of inflammation signs, a fibrous focus was formed. In the patients with lesions at the optic nerve head against the background of the inflammatory reaction subsiding, visual acuity practically recovered. In the patients who received a relapse of the disease against the background of a chronic course of the disease at the remission stage, fresh exudate along the edge of the old 
focus was resorbed within 14 days. Further prophylactic intake of Mercureid brought to a complete block of inflammation signs and restoration of visual acuity to the initial level.

Out of 11 patients with disseminated chorioretinitis, 4 patients had juxtapapillary edema with the formation of a choroidal focus. 2 patients had fresh lesions in the posterior pole and within the paramacular area. 5 patients had exudation along the edge of old lesions. Against the background of the treatment, the phenomena of inflammation were completely blocked, ischemic edema significantly decreased, juxtapapillary foci developed within 1 month, visual acuity recovered. Fresh foci in the posterior pole formed in the form of chorioretinal changes. Exudation along the edge of the old lesions was significantly reduced, while visual acuity did not suffer.

Reviewing the research literature, it was found out that cases of venous and arterial thrombosis develop in more than $30 \%$ of patients with COVID-19. The most common ones are venous thromboembolic conditions $(27 \%)$ [26,27].

In some patients who underwent COVID-19, a spectrum of changes in the retinal vascular system of the eye was marked. The severity of these changes ranged from changes in the diameter of the retinal vessels, which are often accompanied by paravascular edema, to complete occlusion of the vessel [28-35].

In our study, 9 patients had thrombosis of the central retinal vein and its branches. 4 out of these 9 patients got retrombosis of the central retinal vein; the dynamics of visual acuity in this group of patients was negative. In 3 patients with branch thrombosis, there was an ischemic impairment of blood circulation in the optic nerve trunk.

The patients who had eye complications because of long COVID-19, against the background of anti-inflammatory treatment, also received vascular therapy together with Mercureid intake for 1 month. In some patients fundus hemorrhages partially resolved, ischemic edema decreased during one month after the end of the treatment.

\section{Discussion}

The foregoing in this article allows us to conclude the following. As a result of the conducted studies it was found out that in 19 patients (38.7\%) who underwent COVID-19, functional disorders in the form of a light impaired adaptation in 2,3 times against the background of absence of obvious structural changes in the organ of vision at high resolution (visual acuity 1.0) were revealed.

It was caused not only by impaired blood circulation in the eye itself (a decrease in volumetric pulse blood flow by $10 \%$, an increase in vascular tone by 20\%), but in the central part of the visual analyzer (an increase in the tone of large vessels of the basins of the internal carotid artery and vertebrobasilar arteries by $54 \%$ and small vessels - by $12 \%$ ), but also there is 
probability that it was caused by the neuro-toxic effect of the viral agent itself and the phenomena of hypoxemia.

\section{Benefits for the Patient}

A corrective effect (18\% increase) of light-sensitive retina was registered after a month's course of Mercureid intake in 36 patients (73.4\%).

In some patients from the group under control who had rarely recurrent posterior uveitis (relapses no more than 1 time per year), inflammation was activated after COVID-19. Such state required active anti-inflammatory therapy. The most serious cases were observed in the group of patients with lesions of the retinal vascular system: the phenomenon of rethrombosis of both the central retinal vein and its branches, as well as circulatory disorders in the optic nerve trunk - ischemic optic neuropathy with a sharp deterioration in vision. In these patients (patients from group N 3, $\mathrm{n}=14$ ), the combination of anti-inflammatory and vascular drugs together with Mercureid intake made it possible to stabilize the condition, achieve remission, and, in some cases, raise high visual functions. The number of such patients was 7 people $(50.0 \%)$.

The patients from groups N 2 and N 3 while taking Mercureid needed a lower dosage of drugs used in combination therapy; they showed more positive dynamics of state stabilization and recovery.

\section{References}

1. Wu P, Duan F, Luo C, Liu Q, Qu X, Liang L, et al. Characteristics of ocular findings of patients with coronavirus disease 2019 (COVID-19) in Hubei Province, China. JAMA Ophthalmol. 2020;138:575-8.

2. Ling XC, Kang EY, Lin JY, Chen HC, Lai CC, Ma DH, et al. Ocular manifestation, comorbidities and detection of severe acute respiratory syndrome-coronavirus 2 from conjunctivain coronavirus disease 2019: A systematic review and meta-analysis. Taiwan J Ophthalmol. 2020;10(3):153-66.

3. Nayak B, Poddar C, Panigrahi M, Tripathy S, Mishra B. Late manifestation of follicular conjunctivitis in ventilated patient following COVID-19 positive severe pneumonia Indian J Ophthalmol. 2020;68:1675-7.

4. Danthuluri V, Grant MB. Update and recommendations forocular manifestations of COVID-19 in adults and children: A narrative review. Ophthalmol Ther. 2020;9:853-75.

5. Bertoli F, Veritti D, Danese C, Samassa F, Sarao V, Rassu N, et al. Ocular findings in COVID-19 patients: A review of direct manifestations and indirect effects on the eye? J Ophthalmol. 2020;2020:4827304.

6. Varga Z, Flammer AJ, Steiger P, Haberecker M andermatt R, Zinkernagel AS, et al. Endothelial cell infection and endotheliitis in COVID-19. Lancet. 2020;395(10234):1417-8.

7. Hanafi R, Roger PA, Perin B, Kuchcinski G, Deleval N, Dallery F, et al. COVID-19 neurologic complication with CNS vasculitis-like pattern. Am J Neuroradiol. 2020;41(8):1384-7.

8. Valderrama EV, Humbert K, Lord A, Frontera J, Yaghi S. Severe acute respiratory syndrome coronavirus 2 infection and ischemic stroke. Stroke. 2020;51:e124-e1e7.

9. Levi M, Thachil J, Iba T, Levy JH. Coagulation abnormalities and thrombosis in patients with COVID-19. Lancet Haematol. 2020;7(6):e438-40. 
10. Nouri-Vaskeh M, Sharifi A, Khalili N, Zand R, Sharifi A. Dyspneic and non-dyspneic (silent) hypoxemiain COVID-19: Possible neurological mechanism. Clin Neurol Neurosurg. 2020;198:106217.

11. Sheblawi M, Wang K, Viveiros A, Nguyen Q, Zhong JC, Turner AJ, et al. Angiotensin-Converting Enzyme 2: SARS-CoV-2 receptor and regulator of the renin-angiotensin system: Celebrating the 20th anniversary of the discovery of ACE2. CircRes. 2020;126(10):1456-74.

12. Mehra MR, Desai SS, Kuy S, Henry TD, Patel AN. Cardiovascular disease, drug therapy and mortality in COVID-19. N Engl J Med. 2020.

13. Xia W, Shao J, Guo Y, Peng X, Li Z, Hu D. Clinical and CT features in pediatric patients with COVID-19 infection: Different points from adults. Pediatr Pulmonol. 2020.

14. Rawson TM, Moore LSP, Zhu N, Ranganathan N, Skolimowska K, Gilchrist M, et al. Bacterial and fungalcoinfection in individuals with coronavirus: A rapid review to support COVID-19 antimicrobial prescribing. Clin Infect Dis. 2020.

15. Chen N, Zhou M, Dong X, Qu J, Gong F, Han Y, et al. Epidemiological and clinical characteristics of 99 cases of 2019 novel coronavirus pneumoniain Wuhan, China: a descriptive study. Lancet. 2020;395(10223):507-13.

16. Goossens H, Ferech M, Vander Stichele R, Elseviers M, ESAC Project Group. Outpatient antibiotic use in European association with resistance: a cross-national data base study. Lancet. 2005;365(9459):579-87.

17. Llor C, Bjerrum L. Antimicrobial resistance: risk associated with antibiotic overuse and initiatives to reduce the problem. Ther Adv Drug Saf. 2014;5(6):229-41.

18. Struelens MJ. The epidemiology of antimicrobial resistance in hospital acquired infections: problems and possible solutions. BMJ. 1998;317(7159):652-4.

19. Gold DM, Galetta SL. Neuro-ophthalmologic complications of coronavirus disease 2019 (COVID-19). Neurosci Lett. 2021;742:135531.

20. Douglas KA, Douglas VP, Moschos MM. Ocular manifestations of COVID-19 (SARS-CoV-2): A critical review of current literature. In Vivo. 2020;34(3 Suppl):1619-28.

21. Drannik GN, Gorpinchenko II, Kurchenko AI, Gusev SN. Efficacy study of new drug Mercureid (MSC-428) in anti-TNF $\alpha$ therapy for chronic prostatitis. Acta Sci Cancer Biol. 2019;3:9-16.

22. Valerievich LO. Results of multi target therapy anti-pd-1/pd-11/cd19/cd25/cd38 with application of msc- 428 molecules in patients with different oncopathology. Cancerbiology. 2018;2(10).

23. Vladlena G Dubinina. The results of MSC-428 nanomolecules application in neoadjuvant therapy in patients with breast cancer. J Oncopathol Clin Res. 2019;3(1).

24. Velichko LN. Study of the effect of the drug "Mercureid" on the incidence of influenza. Research report Department of Immunology and Pathomorphology the Filatov institute of eye diseases and tissue therapy of the national academy of medical sciences of Ukraine. 2016.

25. Blomberg B, Mohn KG, Brokstad KA, Zhou F, Linchausen DW, Hansen BA, et al. Long COVID in a prospective cohort of home-isolated patients. Nat Med. 2021:1-7.

26. Becker R. COVID-19 update: COVID-19-associated coagulopathy. J Thromb Thrombolysis. 2020.

27. Klok FA, Kruip MJ, Van der Meer NJ, Arbous MS, Gommers DA, Kant KM, et al. Incidence of thrombotic complications in critically ill ICU patients with COVID-19. Thromb Res. 2020;191:145-7.

28. Montesel A, Bucolo C, Mouvet V, Moret E, Eandi CM. Case report: central retinal artery occlusion in a COVID-19 patient. Front Pharmacol. 2020;11:588384.

29. Guégan JP, Legembre P. Nonapoptotic function of FAS/CD95 in the immune response. FEBS J. 2018;285:809-27.

30. Vanden Broek T, Borghans JAM, Van Wijk F. The full spectrum of human naïve T-cells. Nat Rev Immunol. 2018;18:363-73.

31. Sharpe AH, Pauken KE. The diverse functions of the PD1 inhibitory pathway. Nat Rev Immunol. 2018;18:153-67.

32. Schonrich G, Raftery MJ. The PD-1/PD-L1 axis and virus infections: a delicate balance. FronT-cell Infect Microbiol. 2019;13(9):207. 
33. Phelouzat MA, Laforge T, Arbogat A, Quadri RA, Boutet S, Proust JJ. Susceptibility to apoptosis of T lymphocytes from elderly humans is associated with increased in-vivo expression of functional Fas receptors. Mech Ageing Dev. 1997;96:35-46.

34. Zheng HY, Zhang Mi, Yang CX, Zhang N, Wang XC, Yang XP, et al. Elevated exhaustion levels and reduced functional diversity of T-cells in peripheral blood may predict severe progression in COVID-19 patients. Cell Mol Immunol. 2020;17(5):541-3.

35. Crotty S, Kersh EN, Cannons J, Schwartzberg PL, Ahmed R. SAP is required for generating long-term humoral immunity. Nature. 2003;421(6920):282-7. 